This is the author's final, peer-reviewed manuscript as accepted for publication. The publisher-formatted version may be available through the publisher's web site or your institution's library.

\title{
Statistical process control for monitoring nonlinear profiles: a six sigma project on curing process
}

Shing I. Chang, Tzong-Ru Tsai, Dennis K. J. Lin, Shih-Hsiung Chou, \& Yu-Siang Lin

\section{How to cite this manuscript}

If you make reference to this version of the manuscript, use the following information:

Chang, S. I., Tsai, T., Lin, D. K. J., Chou, S., \& Lin, Y. (2012). Statistical process control for monitoring nonlinear profiles: A six sigma project on curing process. Retrieved from http://krex.ksu.edu

\section{Published Version Information}

Citation: Chang, S. I., Tsai, T., Lin, D. K. J., Chou, S., \& Lin, Y. (2012). Statistical process control for monitoring nonlinear profiles: A six sigma project on curing process. Quality Engineering, 24(2), 251-263.

Copyright: Copyright $@$ Taylor \& Francis Group, LLC.

Digital Object Identifier (DOI): doi:10.1080/08982112.2012.641149

Publisher's Link: http://www.tandfonline.com/doi/abs/10.1080/08982112.2012.641149

This item was retrieved from the K-State Research Exchange (K-REx), the institutional repository of Kansas State University. K-REx is available at http://krex.ksu.edu 


\title{
Statistical Process Control for Monitoring Nonlinear Profiles: A Six Sigma Project on Curing Process
}

\author{
Shing I Chang ${ }^{1}$, Tzong-Ru Tsai ${ }^{2}$, Dennis K.J. Lin ${ }^{3}$, Shih-Hsiung Chou ${ }^{1} \&$ Yu-Siang Lin ${ }^{4}$ \\ ${ }^{1}$ Quality Engineering Laboratory, Department of Industrial and Manufacturing Systems Engineering, \\ Kansas State University, USA \\ ${ }^{2}$ Department of Statistics, Tamkang University, Danshui District, New Taipei City 25137 Taiwan \\ ${ }^{3}$ Department of Statistics, Pennsylvania State University, USA \\ ${ }^{4}$ Department of Industrial Management, National Taiwan University of Science and Technology, Taipei, \\ Taiwan
}

\begin{abstract}
Curing duration and target temperature are the most critical process parameters for high- pressure hose products. The air temperature collected in the curing chamber is represented in the form of a profile. A proper statistical process control (SPC) implementation needs to consider both numeric as well as profile quality characteristics. This paper describes a successful six sigma project in the context of statistical engineering for integrating SPC, a statistical method, to the existing practice of engineering process control (EPC) according to science. A case study on a real production curing process is thoroughly investigated. It is shown that the new findings could potentially result in significant energy savings. The solutions provided in this study can be generalized into other curing processes and applications subjected to both EPC and SPC.
\end{abstract}

Key Words: Autoclave, Bi-Plot Chart, DMAIC, Hotelling $T^{2}$ Chart, Profile Analysis.

\section{Introduction and Motivation}

Hoerl and Snee (2010) propose the term statistical engineering defined as "the study of how to best use statistical concepts, methods and tools, and integrate them with information technology and other relevant sciences to generate improved results." In this paper we discuss a six sigma project to implement SPC on high-pressure hose products produced in a factory, $\mathrm{PH}$ Corporation, which is undergoing a lean six sigma transformation. We demonstrate how the proposed SPC solution is integrated into the current engineering process control operation and its quality assurance system in the statistical engineering framework. Through a case study using real-life data, we discuss its potential impact toward continuous improvement.

High-pressure hose products are made out of alternate layers of rubber and metal wires. Toward the end of the production process, various high-pressure hose reels are loaded and cured. A curing process typically consists of a sealed, heated chamber, called an autoclave or vulcanizer. Most autoclaves or vulcanizers are equipped with multiple 
thermocouples inside their chambers and/or parts. The housing that contains an autoclave or vulcanizer is often called the vessel. The most important information gathered from the thermocouples is the air temperature represented as a nonlinear profile during the curing cycle. A programmable logical controller (PLC) is used to control temperature based on sensor readings or a fixed time interval according to a curing recipe. Shown in Figure 1 is a typical air temperature profile divided into three sections heat-up stage, curing stage, and cool-down stage. It is important for the flat section (the curing stage) of this profile to hold for a fixed period of time. The thermocouples are calibrated from time to time so that the measurement error is negligible. The data collection unit of the PLC is capable of collecting multiple temperature readings per second.

A closer examination of the data set reveals that the temperature readings do not change very swiftly. The data presented in Figure 1 are based on temperature readings taken every few seconds. The variables $X_{1}$ to $X_{6}$ are defined in a later section. Due to the confidentiality agreement with $\mathrm{PH}$ Corporation, we altered the numerical values of the temperature and time units. The target air temperature during the curing stage is 500 degrees Fahrenheit and should last for 480 time units.

****** Insert Figure 1. Air Temperature of One Curing Cycle*****

\section{Problems and Challenges}

Under the framework of six sigma, quality and process improvement is most effective on a project-by-project basis. One of the main emphases in statistical engineering addresses the need to identify projects with high impact. $\mathrm{PH}$ Corporation is in its initial stage of experiencing a six sigma transformation. There are many pending projects throughout its factory. One of the process improvement projects that the quality manager and process engineers of $\mathrm{PH}$ Corporation face is implementation of statistical process control on the curing process because all high-pressure hose products go through this critical process before the final quality check is applied. It is very important to study the variability of the curing process and then to maintain its stability. The proposed SPC project would help ensure that the curing production process is under both engineering control and statistical control. When the final quality check identifies potential quality issues, process and quality engineers can rule out the curing process as a cause if a proper SPC procedure is in place. This study demonstrates how statistical thinking and statistical engineering can be integrated into an existing engineering control application.

Process engineers often presume that the curing process must be under control because it is governed led by a PLC according to a recipe used for many years. This argument comes from engineering process control (EPC) instead of SPC. The practice of EPC may keep a malfunctioning process operating for a while before it eventually spirals out of control when a root cause starts to emerge. An SPC implementation combined with an EPC practice can prevent this from happening (see Montgomery et al., 
1994 and Del Castillo, 2002). The cost of scrapping an entire load of cured product is prohibitively high. Therefore, this is one of the high impact projects during the initial stage of six sigma transformation.

The key question concerning process monitoring is: "Is a curing process in control given the air temperature profile generated at the end of a curing cycle?" One of our objectives involves the design and implementation of a SPC plan on this process to make sure that the process is in control in all aspects. A process is assumed to exhibit statistical control when only common cause variability exists in process parameters of the system. If a curing cycle is in control, the quality of products being cured would be satisfactory given that the raw material meets its intended specifications. On the other hand, if a process is out of control, it is very likely products inside the vessel will not be cured properly. The major benefit of an in-control process is product consistency that translates into better product quality and thus customer satisfaction. Reduced waste is also an important benefit.

The current process-monitoring practice involves manual visual inspection of a plot similar to Figure 1 at the end of a curing process. All temperature readings are recorded in a database but a hard copy printout is generated for quality assurance purpose. The operator attending the vessel would examine this plot and decide whether the process is in control or not. If the curing is believed to be successful, a "pass" would be written on the printout. The current curing recipe calls for the following sequence: (1) open the heat steam valve rapidly to heat the chamber air temperature to 500 degrees Fahrenheit, (2) maintain this target temperature for 480 time units, (3) open a valve to vent off the pressure for a fixed period, (4) spray water for a fixed period, (5) rest for a fixed period, (6) spray water a second time for a fixed period, and then (7) open the chamber door to unload the cured products. The impact on temperature for sequence of actions from steps (3) to (7) is summarized in Figure 2. Since the timing and duration for each stage is also controlled by a PLC, operators would glance at the "general pattern" for quality assurance. The current practice is very subjective and operator-dependent. There is a great opportunity to introduce quality monitoring for continuous quality improvement.

\section{Define Critical-to-Quality Characteristics}

In this study, we focus on the air temperature setting considered to be the most critical to a curing process. The current practice involves one curing recipe applied to all highpressured host products regardless of their individual specifications. Each type of hose product requires a minimal period of curing time at a set temperature for the major functional requirement, that is, the proper cross linking of the rubber material. A hose product without a proper curing will fail either in the pressure test or in the field more quickly than expected. In addition, a gradual cooling procedure is designed to maintain the cosmetic requirement on the outside of a hose product, that is, a smooth look without any blistering. It is critical that both aspects are met during the curing production for customer satisfaction. The air temperature profiles are recorded to reflect the production 
quality from batch to batch in term of the most critical process parameter - the air temperature.

After consultation with the quality manager, process engineers, and IT specialist, we define critical-to-quality characteristics (CTQ), often defined in the DMAIC (Define, Measure, Analyze, Improve, and Control) process, as follows:

- QC1: the time to reach the target air temperature 500 degrees Fahrenheit must be swift although the PLC does not specify a time;

- QC2 and QC3: the air temperature must maintain a target value of 500 degrees Fahrenheit (QC2) for a fixed target duration 480 time units (QC3); and

- QC4: the air temperature must be reduced "gradually" according to sequences (3) to $(7)$ defined earlier.

After a curing cycle, an air temperature profile similar to Figure 1 is given. Based on the profile, quality engineers determine whether the CTQs i.e. QC1 to QC4 are in control. A Phase I data set of a curing process was collected over a one month period. Most quality engineers are equipped to implement SPC for QC1 defined above. However, examining Figure 3 during the curing stage, quality engineers were puzzled by multiple types of patterns among 153 profiles related to QC2 and QC3. It is not clear which existing control charting methods could be directly applied for process monitoring purposes.

Another challenge is to implement SPC for QC4 in which numerical standards are illdefined. Quality engineers were overwhelmed by multiple segments and the large amount of data when they examined Figure 4 containing multiple temperature profile segments during the cool-down stage.

${ }^{\star * \star * \star}$ Insert Figure 2 here. A Typical Air Temperature Profile during the Cool-down Stage ${ }^{* * * * *}$

${ }^{* * * *}$ Insert Figure 3. Patterns of the Air Temperature Profiles during the Curing Stage ${ }^{* * *}$

${ }^{* * * *}$ Insert Figure 4. Patterns of the Air Temperature Profiles during the Cool-Down Stage $* * *$

\section{Overview of a System Framework for Data Processing}

In the framework of statistical engineering, it is important to integrate statistical methods and tools into IT practices. Figure 5 shows a proposed system framework related to data processing before SPC can be implemented on the curing process. This framework has four components, i.e., middleware, data warehouse, SPC, and decision making. The process data are first retrieved from various databases corresponding to the vent valve, vulcanizer, and water spray valve. Next, the middleware component provides a data preprocessing function, that is, data loading, data cleaning, transforming, integrating, and refreshing on the raw data. The data warehouse is a repository that stores, arranges and 
organizes information for SPC implementation or any other analysis purposes. Furthermore, the SPC component provides a basic process monitoring for decision making. Currently, the frontend databases are fed by the PLC. Our tasks involve the development of the middleware, SPC and decision making components that will be integrated into the existing quality assurance database.

$\mathrm{PH}$ corporation, like many other manufacturers, are experiencing a modern day factory phenomenon, that is, a data tsunami. Lots of data are generated by various operations, but its use in decision making is ignored. The PLC that controls the curing process is capable of generating a large volume of data, most of which is simply stored, archived, and eventually deleted. None or very little of it is transformed into useful information or statistics on which insightful decision makings can be made. There is really a missing link between the statistical thinking/methods which suggested collecting these data and engineering practice. In this study, we propose to integrate IT and the proposed SPC procedure.

\section{${ }^{* * * *}$ Insert Figure 5. Diagram of System Structure for Data Processing ***}

\section{Establish Measure of Critical-to-Quality Characteristics}

This measure step is the second step of the DMAIC process. Based on the CTQs QC1 to QC4, we would like to establish statistics that can directly measure the CTQs and be fed into control charts. It is straightforward to quantify QC1 as $X_{1}$, which is the duration between the start of a curing stage and the start of the countdown timer of QC3. This statistic is relatively easy to obtain via an algorithm written in Matlab.

It is a challenge to derive statistics based on QC2 and QC3. A closer look at QC2 and QC3 reveals that they are not exactly random variables in the usual sense because a PLC is used to make sure that each cycle achieves the targets for QC2 and QC3, i.e. 500 degrees Fahrenheit for 480 time units. When the air temperature reading gets close to 500 degrees Fahrenheit, the PLC starts the timer for 480 time units to achieve the required fixed duration. Based on the data recording tag shown in the database, the PLC would start the curing timer when a temperature reading first reaches 492 degrees Fahrenheit. Each air temperature profile exhibits a different pattern initially as shown in Figure 3. The temperature would eventually reach the steady state of 500 degrees Fahrenheit. Therefore, the "true" quality characteristic for the curing time requirement is how much time each profile stays at or above 500 degrees Fahrenheit.

We define $X_{2}$ as the cumulative time that temperature readings exceeding the target temperature $(500-\delta)$, where $\delta$ is the standard deviation of the steady-state temperature readings. Note that $\delta$ is small because of the PLC control actions. Based on the steadystate segment of the temperature profiles shown in Figure 3, $\delta$ is estimated to be 0.05 degrees Fahrenheit. This quality characteristic can be obtained by an algorithm written in Matlab to process any profile during the curing stage. It captures the key characteristics important for the curing recipe, i.e. QC2 and QC3. 


\section{A Solution to Profile Analysis during the Cool-down Stage}

We propose the use of profile monitoring techniques for tackling the SPC implementation issues for QC4. Profile monitoring techniques have gained much attention since Jin and Shi (1999) introduced their application to the force of a stamping process. Three general approaches often used to implement SPC on profiles as quality characteristics include: the use of process parameters, the use of projected space, and the use of critical spatial information. Most literature on this topic follows the first category because the process parameters are often independent of each other, which is one of the conditions required to be used for simultaneous charting of univariate control charts (see Kang and Albin, 2000; Kim et al., 2003; Noorossana et al., 2010a and $2010 \mathrm{~b}$ ). The second category is the use of projected space in which original profiles are transformed from one domain into another domain with axes orthogonal to each other. The principal component analysis (Jones and Rice, 1992) is often used for this purpose. Other work in this category includes Vapnik (1998), Walker and Wright (2002), Woodall et al. (2004), and Moguerza et al. (2007). Finally, the third category is the use of spatial information. The core idea is to reduce the number of observations in the original profile but still maintain crucial profile information. Those methods can be found in Grossmann and Morlet (1984), Mallat (1989), Strang and Nguyen (1997), Jin and Shi (1999), Chicken et al.(2009), and Chang and Yadama (2010).

The main activities during the cool-down stage have to do with the proper temperature drops in four segments. Depending on the load i.e. heat mass in the vessel, these temperature profiles exhibit different characteristics but hold the general patterns as shown in Figure 4. The geometric shapes from various cycles deviate from each other greatly in each segment. The steepest temperature drops usually take place during the segments of vent opening and spraying. This pattern will break if the vent does not open or the water tank runs out of water during a spraying cycle. Instead of using one model for these complicated profiles at this stage, it would be much easier to break this stage into smaller segments according to the PLC recipe. The current recipe contains several fixed intervals - venting, water spray cycle 1 , resting, and water spray cycle 2 . The last segment (not labeled) in Figure 2 is not important because a load may sit in the vessel for a period of time before it is unloaded. The length of this period depends on factory schedules.

We propose statistics $X_{3}, X_{4}, X_{5}$, and $X_{6}$ as the average of deviations from the nominal profiles for each segment for the cool-down steps (3) through (6), respectively. These statistics are chosen to reflect the general trend of the cool-down process. Each statistic identifies the proper behavior within a functional segment so that it is easy for operators to diagnose problematic profiles. As shown in Figure 2, $X_{3}$ to $X_{6}$ are derived from air temperature readings $y_{i j}$ where the index $i$ is defined according to the cool-down recipe and $j=3,4,5,6$. Specifically, the index $i$ of $y_{i j}$ takes the integer values in [1,60], [61, 88], [89, 116], and $[117,140]$ respectively depending on $j=3,4,5$, or 6 .

Process engineers do not know the shape of the nominal profile of the air temperature during the cool-down cycle. We need to estimate it from a Phase I data set. Similar to 
the practice reported by Chang and Yadama (2010) $X_{3}$ to $X_{6}$ can be obtained from the following function:

$$
X_{j}=\frac{\sum_{i=1}^{n_{j}}\left|y_{i j}-\bar{y}_{j}\right|}{n_{j}} j=3,4,5,6 .
$$

where $y_{i j}$ is the $i$ th data point of the $j$ th profile segment, $n_{j}$ is the number of points within segment $j$, and $\bar{y}_{j}$ is the $j$ th segment of the "average" profile fitted over all profiles in a Phase I data set. Judging from Figures 2 and 4 , linear functions should be sufficient to model $\bar{y}_{j}$, for $j=4,5$ and 6 but perhaps not for $j=3$. In this study, we choose to use a B-spline function to fit each profile segment because it is capable of fitting either linear or nonlinear profiles. For details of B-spline fitting, please refer to Mortenson (2006).

\section{Analyze Curing Process via Control Charting}

The third step of the DMAIC process involves the use of a Phase I study of control charts to understand causes of variation and identify potential root causes. One approach for implementing SPC combines $X_{1}$ to $X_{6}$ into one multivariate control chart. This design allows the monitoring of the curing process in a single chart instead of spreading the task into six individual control charts. However, this approach will delay fault diagnosis when a process is out of control. It would need the involvement of a quality engineer to examine the individual data of $X_{1}$ to $X_{6}$ to identify which variables are responsible for an out-of-control signal. On the other hand, the approach of using six individual control charts simultaneously would increase the overall type I error of control charting. Moreover, the correlation information between quality characteristics would be ignored so that certain out-of-control causes would not be detected under this approach.

From examining QC1 to QC4 and various stages of curing, we propose a solution to separate variables $X_{1}$ to $X_{6}$ into two groups. The first group consisting of $X_{1}$ and $X_{2}$ is used for monitoring the heat-up and curing stages because both pieces of information are maintained by the heating valve operation. These two variables are highly related because control actions on the heat valve dictate them. The second group consisting of the rest of the variables is mainly used for the cool-down stage. Two multivariate control charts for individual observations are proposed for these two groups, respectively. In this case, each observation corresponds to a curing cycle. In other words, we would treat each cycle as one sample with one set of $X_{1}$ to $X_{6}$ values which are monitored via two control charts.

Possible multivariate control charts for individual observations include Hotelling $T^{2}$ charts (Hotelling, 1947) and MEWMA charts (see Lowry and Montgomery, 1995 and Tracy et al., 1992). A control chart for individual observations is used because each curing cycle only provides one air-temperature profile. Note that the underlying process is controlled by a PLC. Within each cycle it is possible that temperature observations may exhibit 
autocorrelation. However, points on a control chart are plotted from cycle to cycle. Autocorrelation, if it exists, may diminish.

\section{A Case Study - the Curing Process Revisited}

In this study, 153 air temperature profiles (or cycles) of Phase I data are examined to seek opportunities for improvement. Quality and process engineers deemed that they are in control. Each profile is divided into two segments. The first segment aims for the monitoring of heat-up and curing duration, while the second segment is for the monitoring of the cool-down stage. In the first segment, there are two quality characteristics of interest. Both have the same unit, i.e., time duration. The second segment of a cycle contains four quality characteristics of interest, which are related to the average deviations from their nominal nonlinear profiles. Using conventional SPC procedure, we first apply two Hotelling's $T^{2}$ control charts to explore the first segment and second segment on all profiles, respectively. Although the quality engineers claimed that the 153 profiles in Phase I data set are all in control, Hotelling's $T^{2}$ control charts may show different results. In order to establish the control charts for Phase II monitoring, it is necessary to screen out abnormal observations. All plotted points should fall within the control limits. Otherwise, we should seek for explanations and justifications for any out-of-control point to be included. In order to maintain a combined type I error of 0.005 , we establish the control limit for each $T^{2}$ control chart accordingly. Results of Phase I studies for both $T^{2}$ control charts are discussed in the following sections.

\section{Study during the Heat-up and Curing Stage}

We first plot all standardized observations on the Hotelling $T^{2}$ control chart with individual observations (see Vargas, 2003 and Williams et al., 2006). As shown in Figure 6, there are 11 points outside the control limit. We further examine the causes that make these points "abnormal" before any point is discarded from the Phase I data set. Figure 7 shows a bi-plot that plots all points in a two-dimensional plot between $X_{1}$, the heat-up duration, and $X_{2}$, the curing duration above the target 500 Fahrenheit. We draw the control limits of the IX chart (i.e. X-bar chart on individual observations) for $X_{1}$ as well as the lower specification limit 360 time units for $X_{2}$ on Figure 7 . Note that this lower specification is more important than the control limits in that rubber products require a minimal time at a set temperature for proper curing. In this case, the target curing time is set at 480 time units so that most curing cycles would have at least 360 time units above the cured temperature of 500 degrees Fahrenheit. The group of out-of-control points including 23, 77, and 126 indicates that these three cycles take a long time to reach the target curing temperature but their curing duration above 500 degrees Fahrenheit are above the mean curing duration. On the other hand, the group of out-of-control cycles including 1, 9 and 98 shows that the time to reach the target temperature is shorter than that of most cycles. Specifically, cycles 1 and 98 are below the mean curing duration but above the minimal 360 time units. Another cycle 54 also belongs to this group. Although this point falls within the IX chart control limits and is above 360 time units, it is far from the mean point of the ellipsoid causing it to be plotted outside the control limit of the $T^{2}$ 
chart. We keep the cycles of these two groups when establishing the control limits of the $T^{2}$ chart.

The group of out-of-control points that cause concerns consists of cycles $60,73,131$, and 137. Although they reach the target temperature like the majority of the other cycles, they fail to keep the minimal of 360 time units of curing time above 500 degrees Fahrenheit. This result is forwarded to process engineers for more detailed study. After discarding the cycles of this group from the Phase I data set, the new control limit of the Hotelling $T^{2}$ control chart changes from 11.594 to 11.565 (based on Tracy et al., 1992).

${ }^{* * * * *}$ Insert Figure 6 Hotelling's $T^{2}$ Control Chart for $X_{1}$ and $X_{2}{ }^{* * * * *}$

${ }^{* * * * *}$ Insert Figure 7 Bi-plot for $X_{1}$ and $X_{2}{ }^{* * * * *}$

\section{Study during the Cool-down Stage}

The Hotelling's $T^{2}$ control chart for the cool-down stage has four variables $X_{3}$ to $X_{6}$, which are the average deviation statistics from the B-Spline fitted nominal profile. This is displayed in Figure 8. Cycles 41, 136 and 141 are above the control limit of 15.764 . Figure 9 shows detailed plots of each segment with these three profiles highlighted. In various segments, the shapes of these cycles are very different from the rest of the profiles. In addition, we can also use a matrix plot to show the correlation structure among $X_{3}$ to $X_{6}$. As shown in Figure 10, the outliers correspond to cycles 41, 136 and 141 in various places. After removing these points, the control limit of the Hotelling's $T^{2}$ control chart changes from 15.764 to 15.732 .

${ }^{* * * * *}$ Insert Figure 8 Hotelling's $T^{2}$ Control Chart for $X_{3}$ to $X_{6}{ }^{* * * * *}$

*****Insert Figure 9 Cool-down Process Profiles by Segment (Out-of-control Profiles 41, $136,141)^{\star * * * *}$

${ }^{* * * * *}$ Insert Figure 10 Matrix Plot of $X_{3}$ to $X_{6}{ }^{* * * * *}$

\section{Summary of the SPC Tools Used in this Study}

The paradigm of statistical engineering links statistical methods and tools at the operational level to statistical thinking at the strategic level. In this study, various statistical methods integrated with IT tools have been adopted to fulfill this goal. The main idea of statistical thinking in this case is to integrate the statistical process control of the curing process to the current practice which is operated under the engineering control. The implementation of SPC will prevent major process catastrophes from taking place and provide useful information and statistics for informative decision making.

We now summarize how all the statistical methods and tools are used in this study. The main process monitoring tools proposed are a pair of Hotelling $T^{2}$ control charts. The studies described above show how the control limits for both charts are established based on the phase I data set. During the process monitoring phase, an algorithm 
written in Matlab is used to obtain the quality characteristics $X_{1}$ to $X_{6}$ from a sample temperature profile. Then variables $X_{1}$ and $X_{2}$ form an input vector to be used in the first $T^{2}$ control chart as shown in Figure 6 while the rest of the variables are put into the $2^{\text {nd }} T^{2}$ control chart as shown in Figure 8. If the points plotted on both $T^{2}$ control charts fall below their respective control limits, the process is deemed in control. Otherwise, a biplot shown in Figure 7 is used on variables $X_{1}$ and $X_{2}$ for fault diagnoses when the first $T^{2}$ control chart triggered the out-of-control signal. Furthermore, a matrix plot of cooldown variables $X_{3}$ to $X_{6}$ should be used when the second $T^{2}$ control chart triggered the out-of-control signal. Operators are responsible for monitoring both $T^{2}$ control charts at the end of each curing cycle, while quality engineers should use either a bi-plot or a matrix plot for fault diagnoses when an out-of-control signal is triggered by either or both of the $T^{2}$ control charts. During a routine curing operation, operators would use the control charts to judge whether the process is in control or not. When a point plots outside the control limits of either one of the $T^{2}$ control charts, process engineers are then notified to diagnose the process.

\section{Improve the Curing Process}

During the fourth step of the DMAIC process, we identify and generate potential solutions for process improvement. Currently air temperature profiles are not used for any process related analysis or diagnosis. The $\mathrm{PH}$ Corporation will benefit from decision making based on objective data analysis as demonstrated in this case study. Furthermore, during this Phase I study, we have identified a process adjustment opportunity that may potentially save production cost. The distribution of the curing duration above the target temperature reveals that it is possible to trim the target duration to a smaller value as shown in Figure 7. The curing recipe specifies 480 time units of curing to accommodate all types of products so that the actual curing time above 500 Fahrenheit may be at the minimum of 360 time units. Most $X_{2}$ values in Figure 7 are actually above 380 time units. A gap of 20 time units between 360 and 380 can be trimmed from the current setting of 480 time units and most actual curing times would still meet the minimal requirement. As energy costs rise, this savings opportunity may dramatically improve the cost of production while satisfying functional requirements. As a numerical example, for every 200 batches of hose products cured, reducing the curing time by 20 time units will yield an additional $20 \times 200 /(480-20)=8.7$ batches without using additional energy.

Historically, only one curing recipe is used regardless of the type of hose products loaded. It is understandable that the current curing time has to be set at the maximum level to satisfy the worst case scenarios. However, with the $X_{2}$ data analyzed we have demonstrated that this one-size-fit-all practice may be revised to satisfy functional requirements and reduce energy consumption. 


\section{Control the Curing Process by Embedding the Proposed SPC Solution into the Current Quality Assurance Practice}

The final step of the DMAIC process involves the integration of the proposed SPC implementation into the ongoing work flow as shown in Figure 11. A simplified process flow shows that the semi-finished hose products are loaded into a vulcanizer after all braiding processes are finished. Two major subcomponents in the curing process consist of the proposed division of the heat-up/curing process and the cool-down process. Cured products would then go through quality assurance via acceptance sampling before they are shipped to customers.

The shaded box in the middle of Figure 11 summarizes the proposed SPC implementation. In order to gain long-term success of the proposed system, the proposed SPC solutions should be integrated with the existing quality assurance system. This is another example of integrating statistical tools into IT to maintain the gains achieved in the statistical engineering paradigm. As shown in the dashed line in Figure 11, we recommend the inclusion of the proposed outputs to be stored in the existing quality assurance database (QA DB). The solid line connecting to the QA DB represents the existing IT system used for quality assurance while the dash line is the proposed one. Depending on the results of on-line monitoring, i.e., Hotelling $T^{2}$ charts or bi-plot, quality engineers may be able to use this information to decide proper sample sizes for destructive tests or to pay more attention to batches with large variations, for example.

\section{Concluding Remarks}

Following the DAMIC process commonly used in a six sigma project and the paradigm of statistical engineering, an SPC implementation solution is introduced here for a production curing process where there is a mixture of quality characteristics presented in the form of a temperature profile. Using both computer algorithms (non-statistical techniques) and profile monitoring techniques (statistical techniques), we propose a solution to combine critical quality characteristics and profile monitoring into one SPC framework. A Phase I data set is used to demonstrate how the proposed framework involving the use of two Hotelling $T^{2}$ charts is implemented. This case study on curing production process is shown to be successful. The proposed SPC framework is recommended to be integrated into the existing quality assurance process to ensure long-term success. Although the results of this study are based on the vulcanizer of the $\mathrm{PH}$ Corporation, it is straightforward to generalize them into other curing processes. The mechanism and control used in various autoclaves are very similar. In addition, the proposed SPC procedure can be more broadly applied to other EPC/SPC applications. The key of a successful integration of EPC and SPC relies on the transformation from EPC variables into proper quality characteristics for SPC. In this case study, the EPC variables are the curing recipe - 500 degrees Fahrenheit for 480 time units. We were able to convert it into variable $\mathrm{X}_{2}$.

Based on the Phase I study, one important issue that we explored was the cost aspect. The opportunity for improvement lies in the curing recipe. The distribution of the curing 
duration above the target temperature reveals that it is possible to trim the target duration to a smaller value (the current setting is at 480 time units). As energy costs rise, it should be noted and emphasized that there is a great opportunity for cost saving while satisfying the functional requirements.

Although this study only focuses on SPC implementation strategy, further investigations that take the full advantage of the entire set of collected data may be considered. For example, there are other profiles-such as pressure and condensation water temperature-generated from the same process that can potentially improve the stability of the curing process. It is a challenge to incorporate all of them into the process monitoring framework. Future six sigma projects should be considered.

\section{Acknowledgements}

We are grateful to the guest editors and two referees whose constructive comments were extremely helpful.

\section{References}

Chang, S. I., and Yadama, S. (2010). Statistical Process Control for Monitoring NonLinear Profiles Using Wavelet Filtering and B-Spline Approximation. International Journal of Production Research 48(4): 1049-1068.

Chicken, E. and Pignatiello, J.J. Jr, and Simpson, J. (2009). Statistical Process Monitoring of Nonlinear Profiles Using Wavelets. Journal of Quality Technology 41(2): 198-211.

Del Castillo, E. (2002). Statistical Process Adjustment for Quality Control. Wiley, NY.

Grossmann, A. and Morlet, J. (1984). Decompostion of Hardy Functions into Square Integrable Wavelets of Constant Shape. SIAM J. Math. Anal. 15(4), 723-736.

Hoerl, R. W. and Sneed, R. D. (2010). Closing the Gap - Statistical Engineering Links Statistical Thinking, Methods, Tools. Quality Progress, May: 52-53.

Hotelling, H. (1947). Multivariate Quality Control. In C. Eisenhart, M. W. Hastay, and W. A. Wallis, eds. Techniques of Statistical Analysis. New York: McGraw-Hill.

Jin, J., and Shi, J. (1999). Feature-Preserving Data Compression Of Stamping Tonnage Information Using Wavelets. Technometrics 41(4): 327-339.

Jones, M.C. and Rice, J.A. (1992). Displaying the Important Features of Large Collections of Similar Curves. The American Statistician 46(2): 140-145.

Kang, L., and Albin, S. L. (2000). On-Line Monitoring When the Process Yields a Linear Profile. Journal of Quality Technology 32(4): 418-426. 
Kim, K., Mahmoud, M. A., and Woodall, W. H. (2003). On the Monitoring of Linear Profiles. Journal of Quality Technology 35(3): 317-328.

Lowry, C. A. and Montgomery, D. C. (1995). A Review of Multivariate Control charts. IIE Transactions 27(6): 800-810.

Mallat, S. (1989). A Theory for Multiresolution Signal Decomposition: The wavelet Representation. IEEE Transactions on Pattern Analysis and Machine Intelligence 11(7): 674-693.

Moguerza, J.M., Munoz, A, Psarakis, S. (2007). Monitoring Nonlinear Profiles using Support Vector Machine. Lecture Notes in Computer Science 4756: 574-583.

Montgomery, D. C. (2009). Introduction to Statistical Quality Control. $6^{\text {th }}$ Edition. New York, NY: John Wiley \& Sons, Inc..

Montgomery, D. C., Keats, J. B., Runger, G. C., and Messina, W. S. (1994). Integrating Statistical Process Control and Engineering Process Control. Journal of Quality Technology, 26 (2), 79-87.

Mortenson, M. E., C. (2006). Geometric Modeling. New York, NY: Industrial Press Inc.

Noorossana, R. Eyvazian, M., Amiri, A and Mahmoud, M. A. (2010). Statistical Monitoring of Multivariate Multiple Linear Regression Profiles in Phase I with Calibration Application. Quality and Reliability Engineering International 26(3): 291-303.

Noorossana, R. Eyvazian, M. and Vaghefi, A. (2010). Phase II Monitoring of Multivariate Simple Linear Profiles. Computers and Industrial Engineering 58(4): 563-570.

Strang, G. and Nguyen, T. (1997). Wavelets and filter Banks, Revised Edition, Wellesley, MA: Wellesley-Cambridge Press.

Tracy N. D., Young, J. C. and Mason, R. L. (1992). Multivariate Control Charts for Individual Observations. Journal of Quality Technology 24(2): 88-95.

Vapnik, V. N. (1998). Statistical Learning Theory. New York, NY: Wiley.

Vargas N. J. A. (2003). Robust estimation in multivariate control charts for individual observations. Journal of Quality Technology 35(4): 367-376.

Williams, J. D., Woodall, W. H., Birch, J. B. and Sullivan, J. H. (2006). Distribution of Hotelling's $T^{2}$ Statistic Based on the Successive Difference Estimator. Journal of Quality Technology 38(3): 217-229.

Woodall, W. H., Spitzner, D. J., Montgomery, D. C., and Gupta, S. (2004). Using Control Charts to Monitor Process and Product Quality Profiles. Journal of Quality Technology 36(3): 309-320.

Walker, E. and Wright, S. P. (2002). Comparing Curves Using Additive Models. Journal of Quality Technology 34(1): 118-129. 


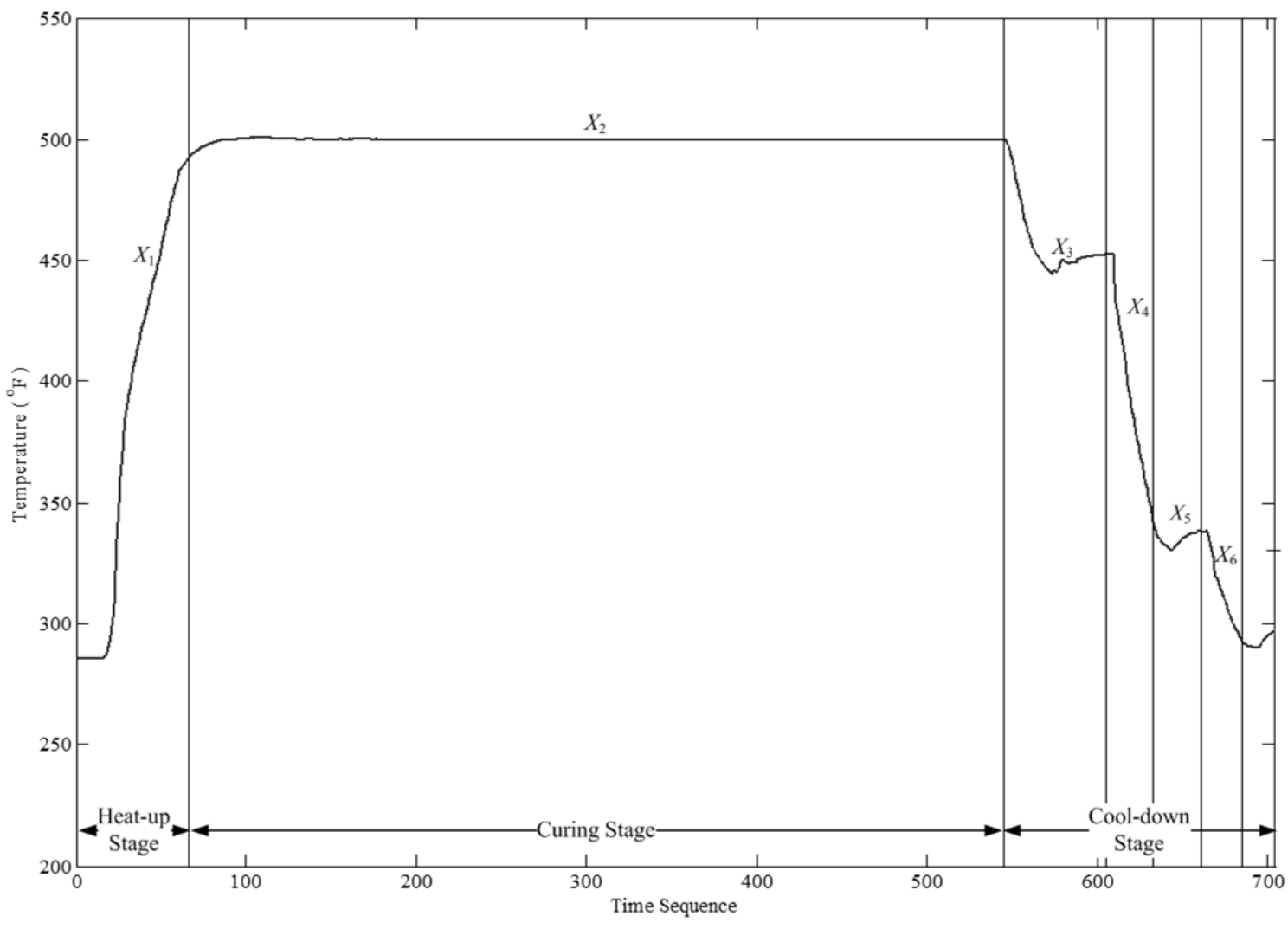

Figure 1. Air Temperature Profile of a Typical Curing Cycle 


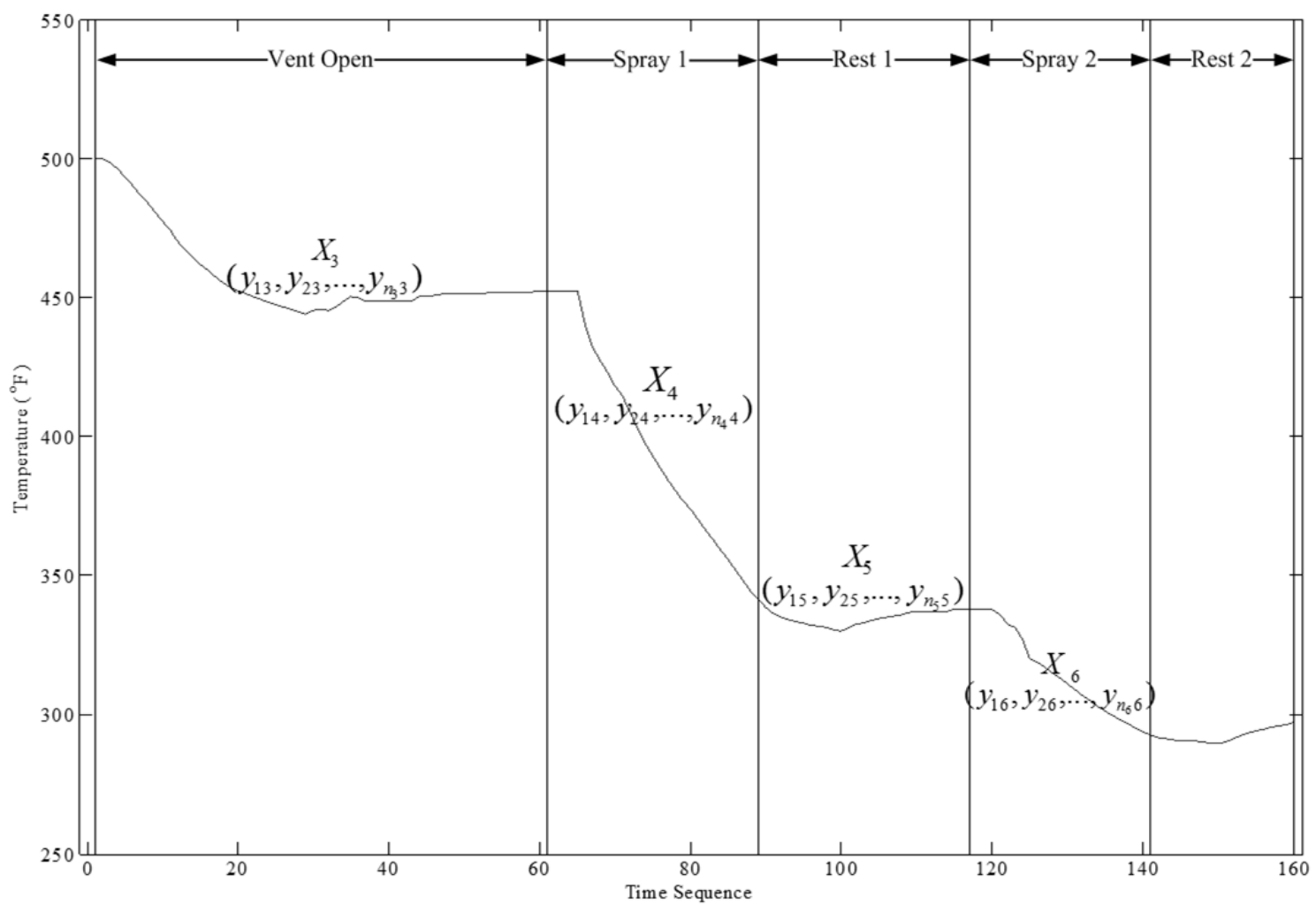

Figure 2. A Typical Air Temperature Profile during the Cool-down Stage

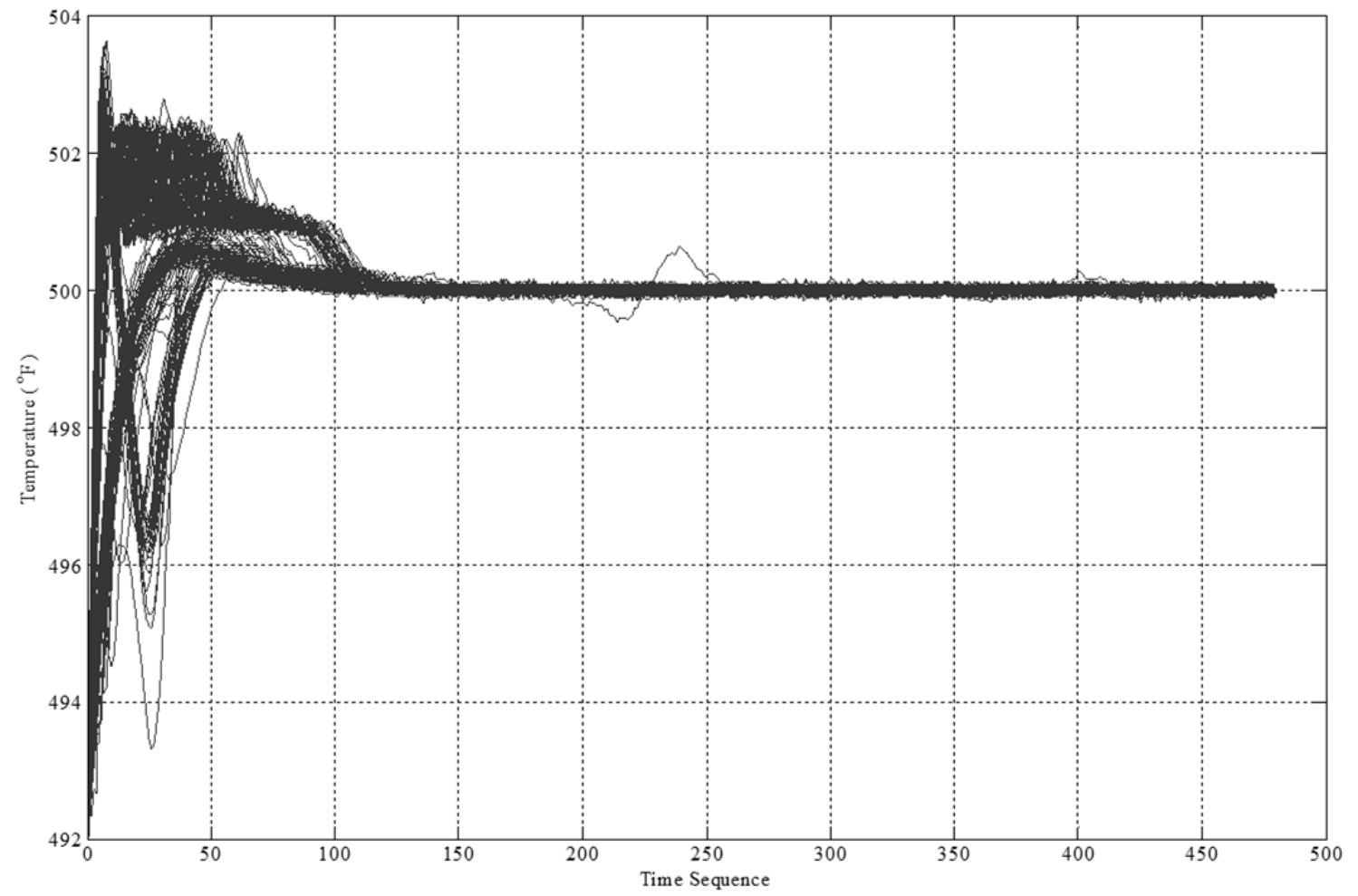

Figure 3. Patterns of the Air Temperature Profiles during the Curing Stage 


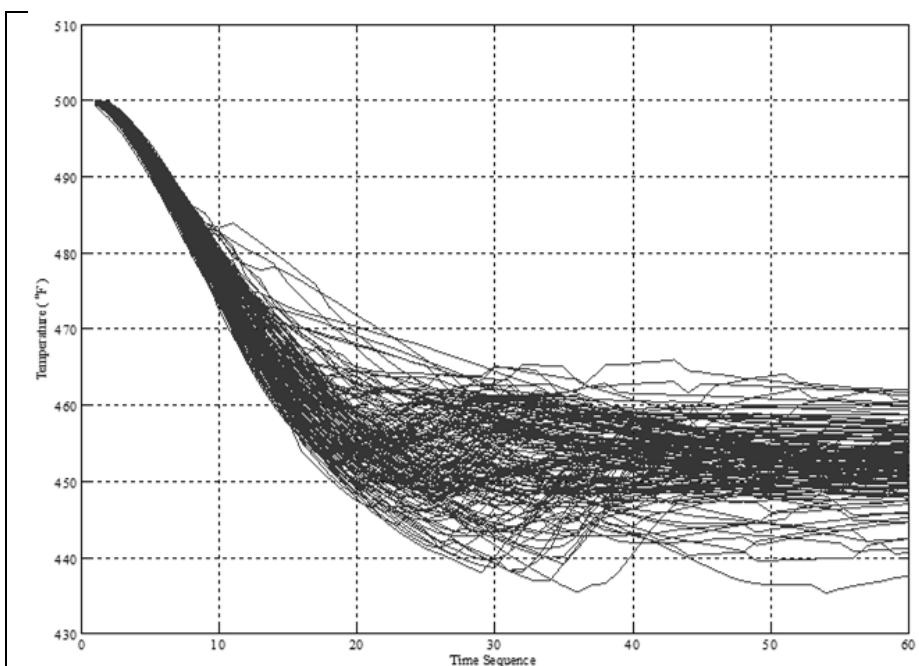

(a) The duration of Vent open $\left(X_{3}\right)$.

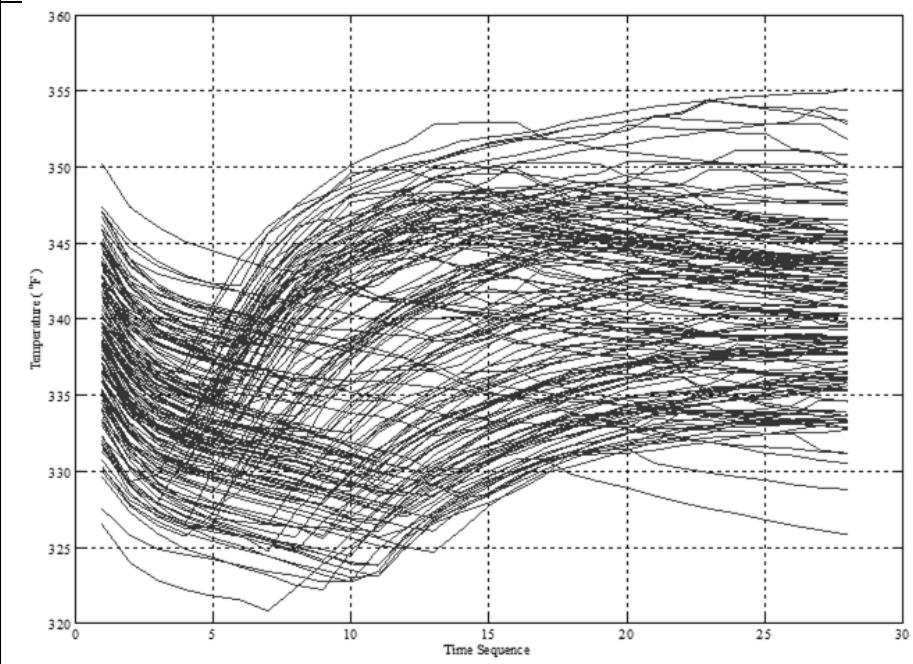

(c) The duration of Rest $1\left(X_{5}\right)$.

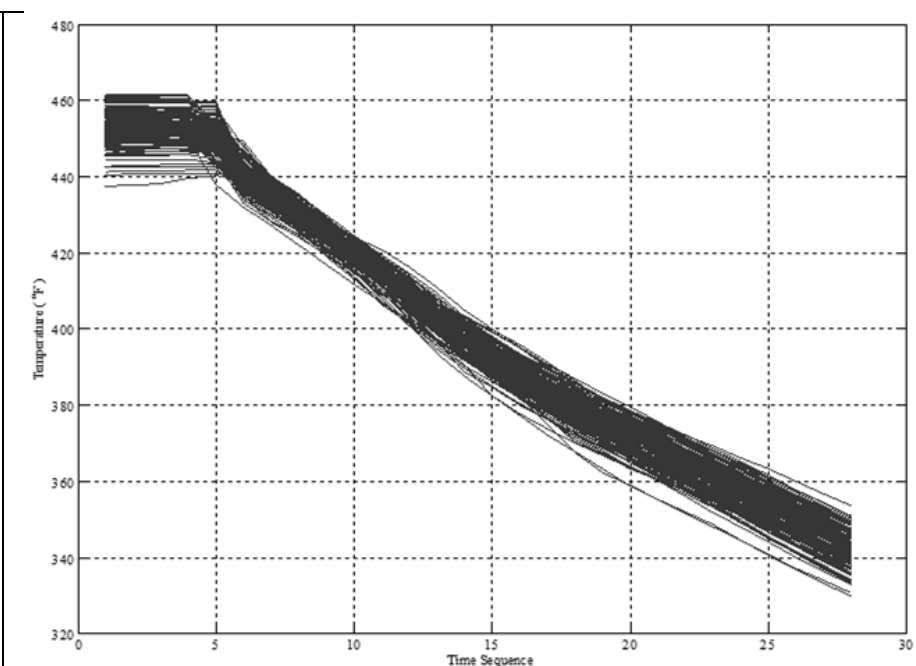

(b) The duration of Spray $1\left(X_{4}\right)$.

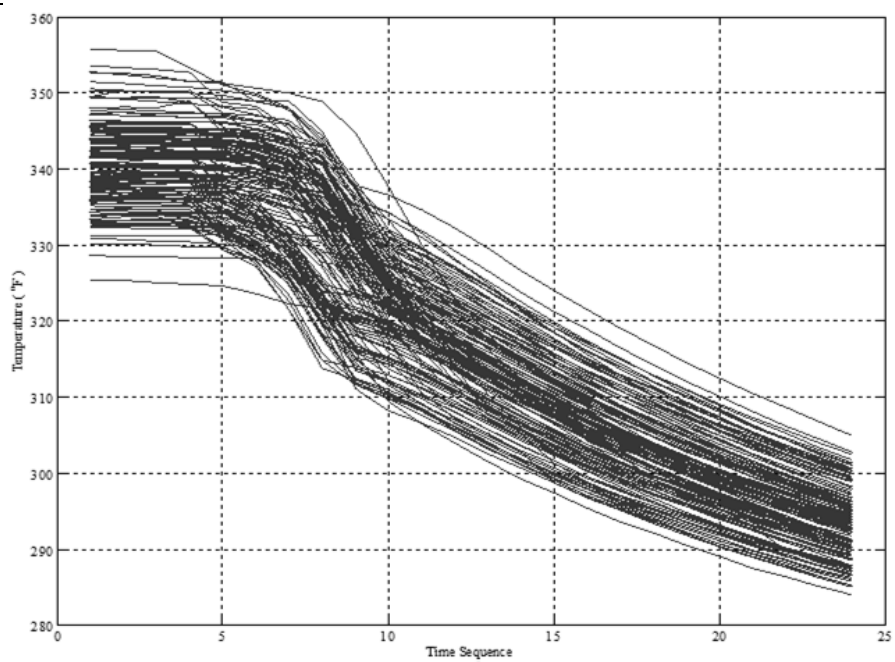

(d) The duration of Spray $2\left(X_{6}\right)$.

Figure 4. Patterns of the Air Temperature Profiles during the Cool-Down Stage 


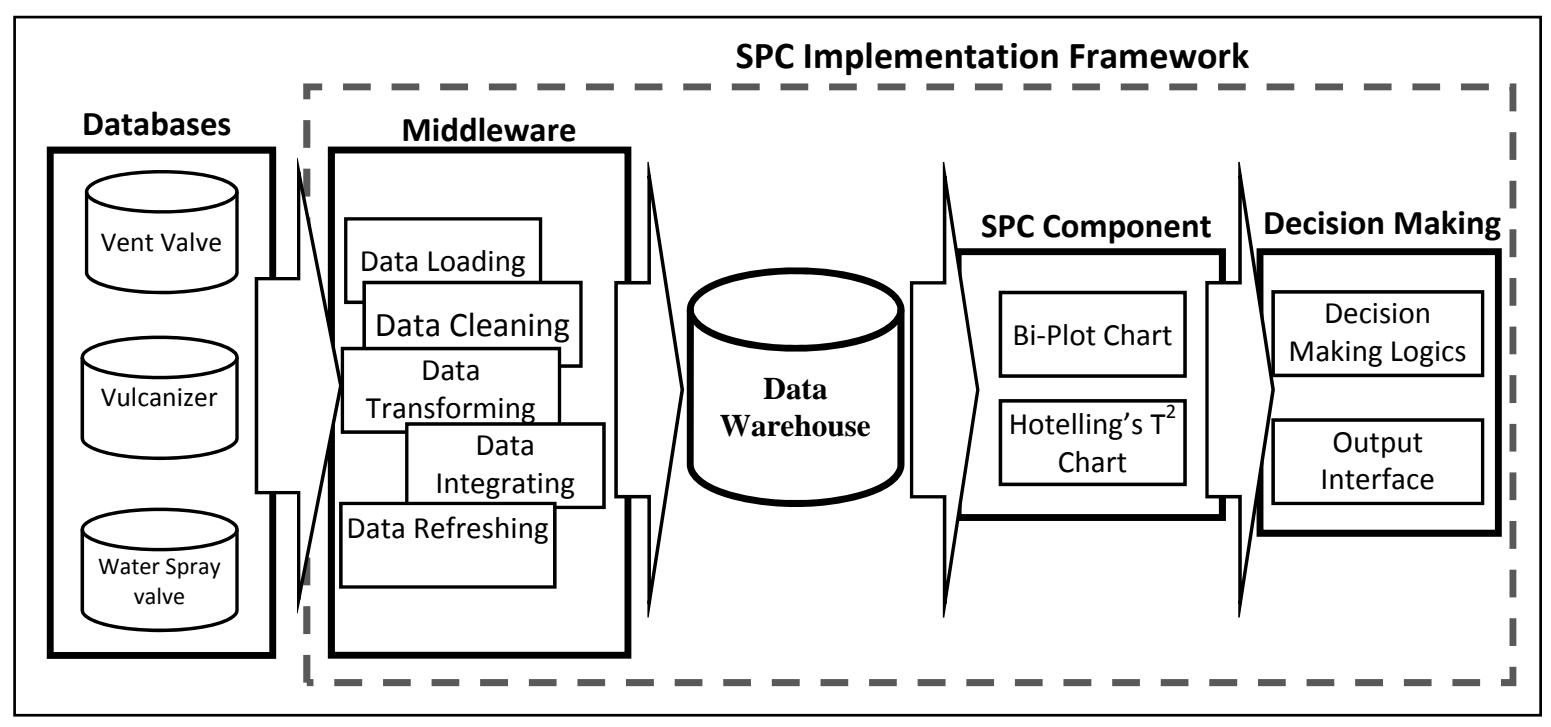

Figure 5. Diagram of System Framework for Data Processing.

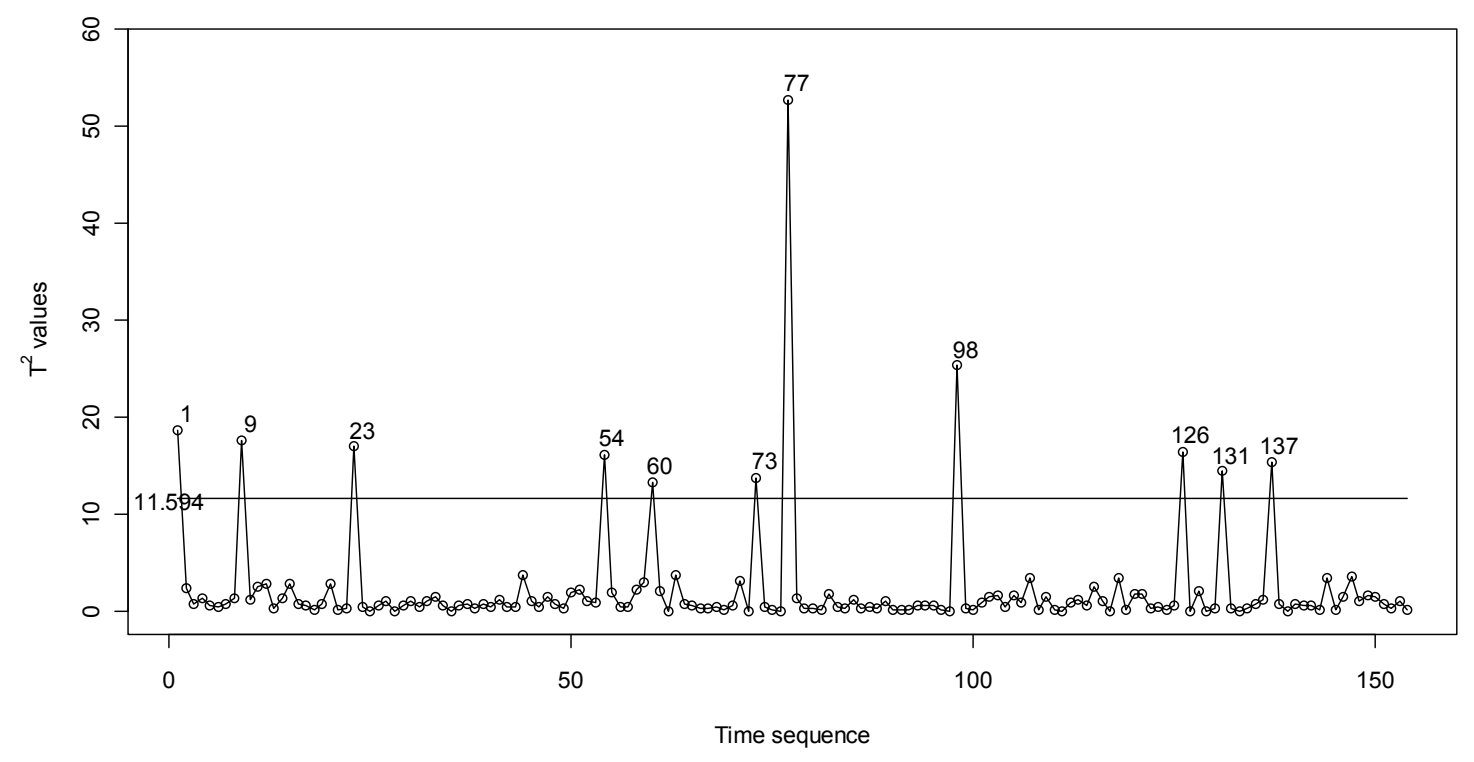

Figure 6. Hotelling $T^{2}$ control chart for $X_{1}$ and $X_{2}$ in the Heat-up and Curing Stage 


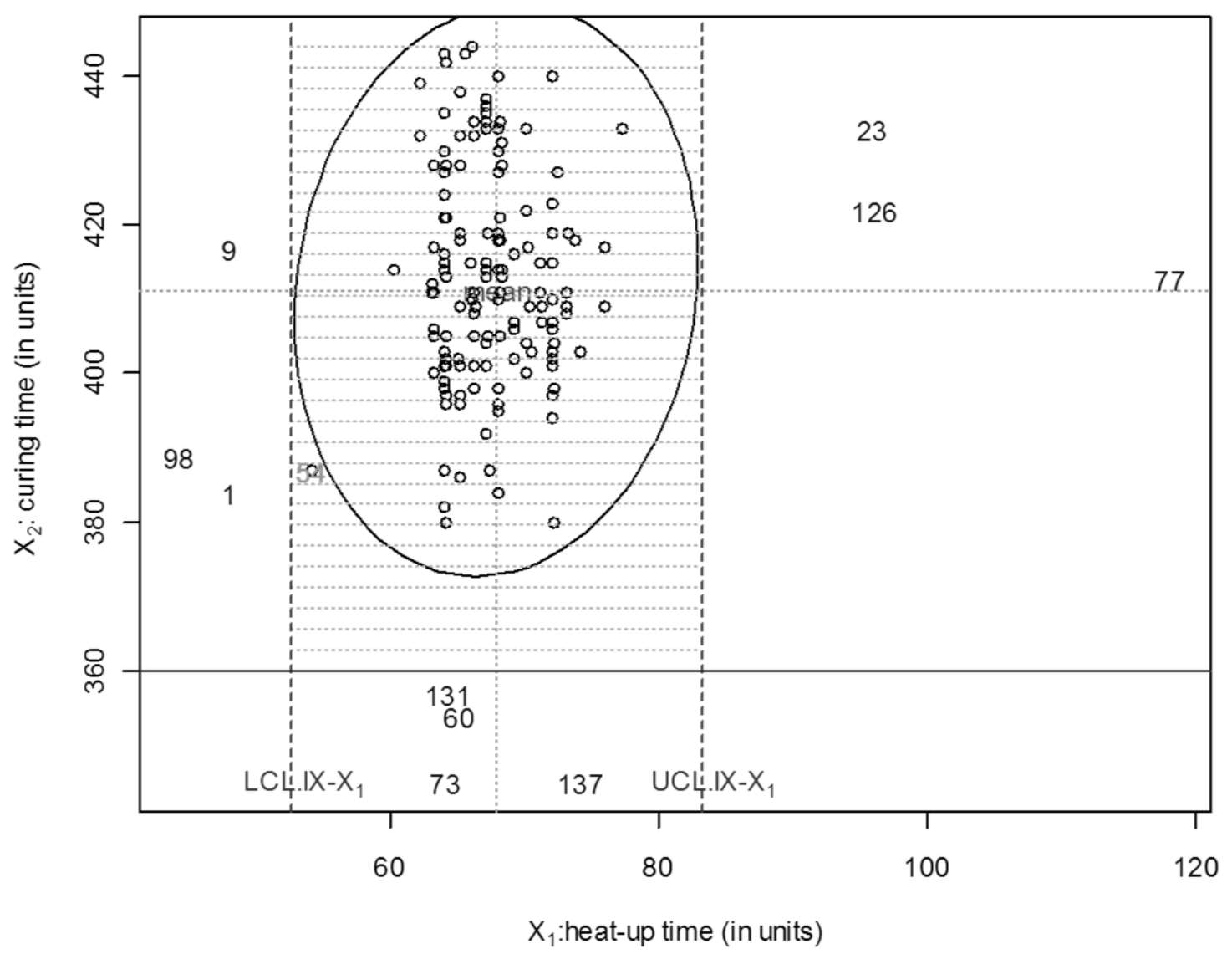

Figure 7. Bi-plot and IX Control Limits for Heat-up Time and Curing Time

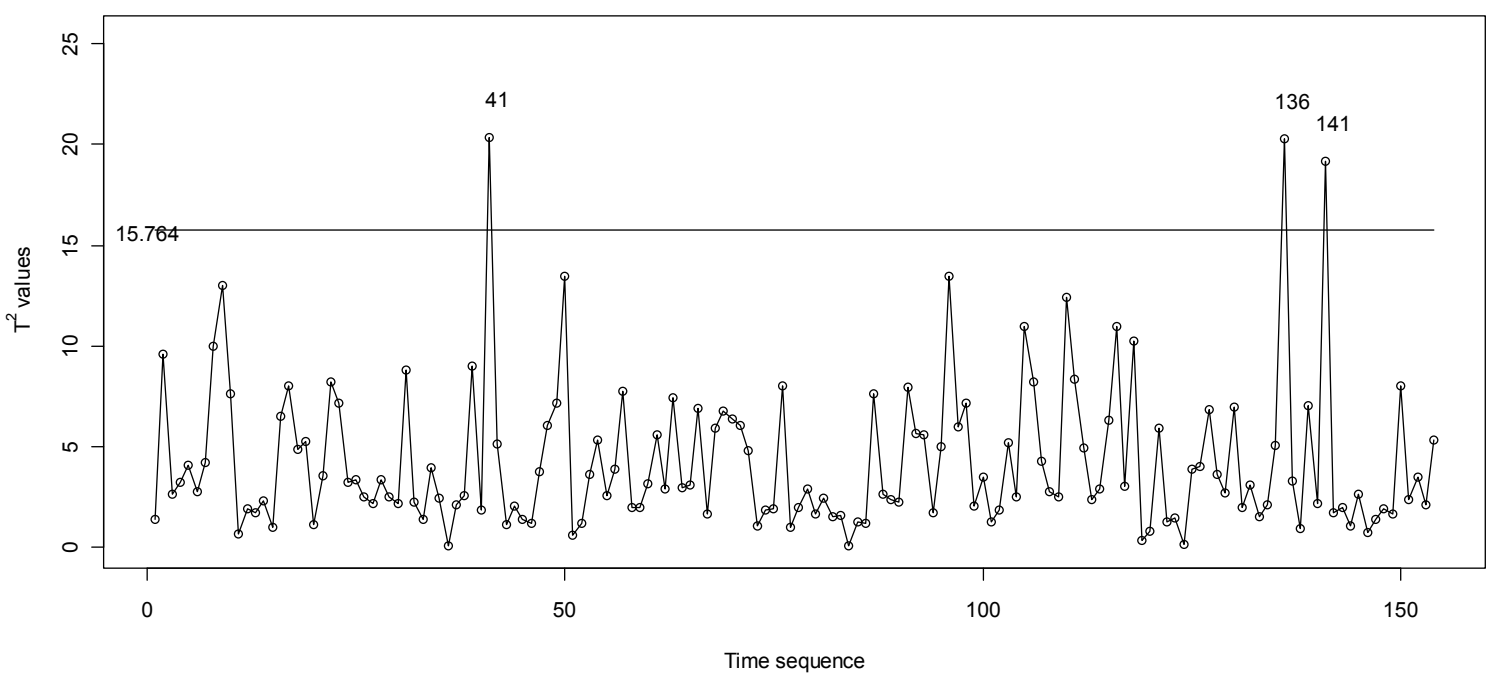

Figure 8. Hotelling $T^{2}$ Control Chart for Cool-Down Variables $X_{3}$ to $X_{6}$ 


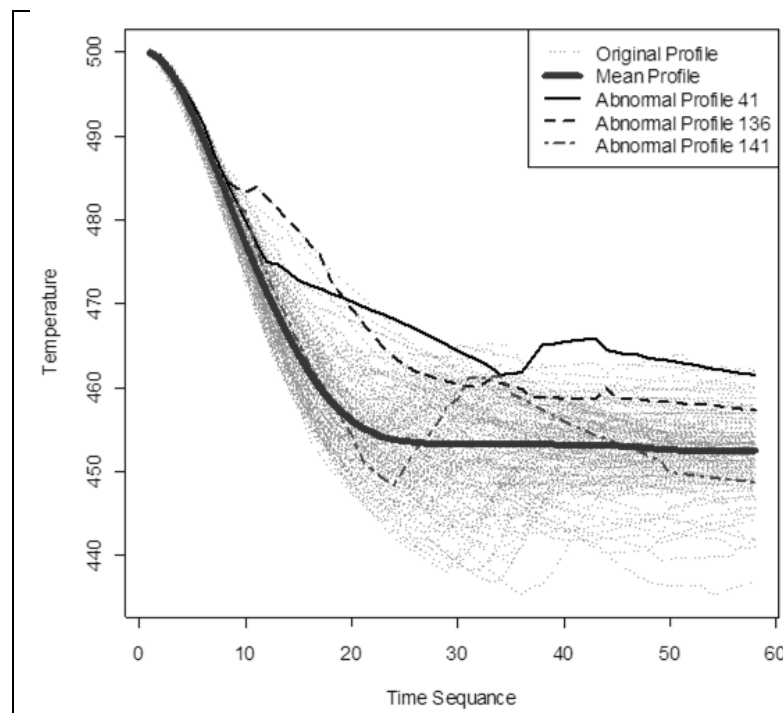

(a)

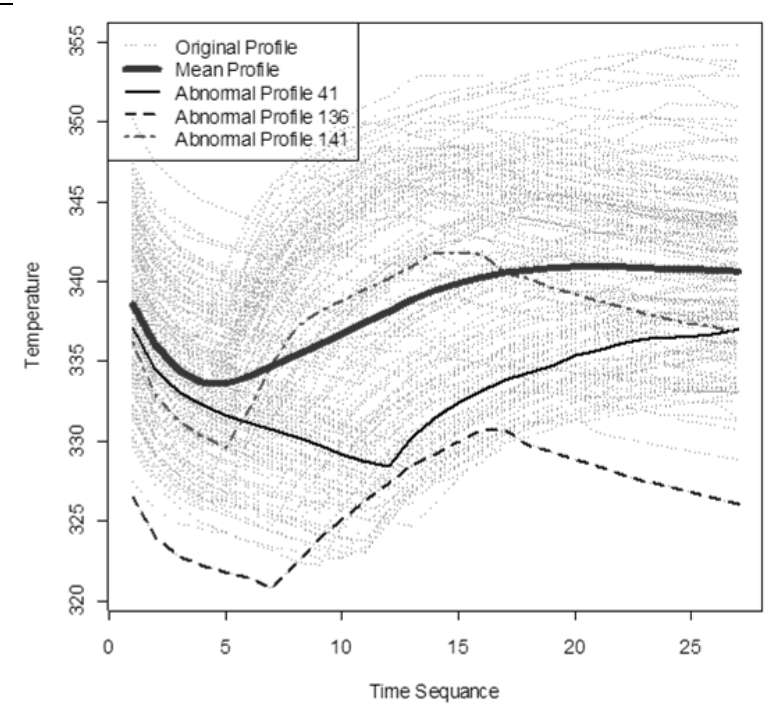

(c)

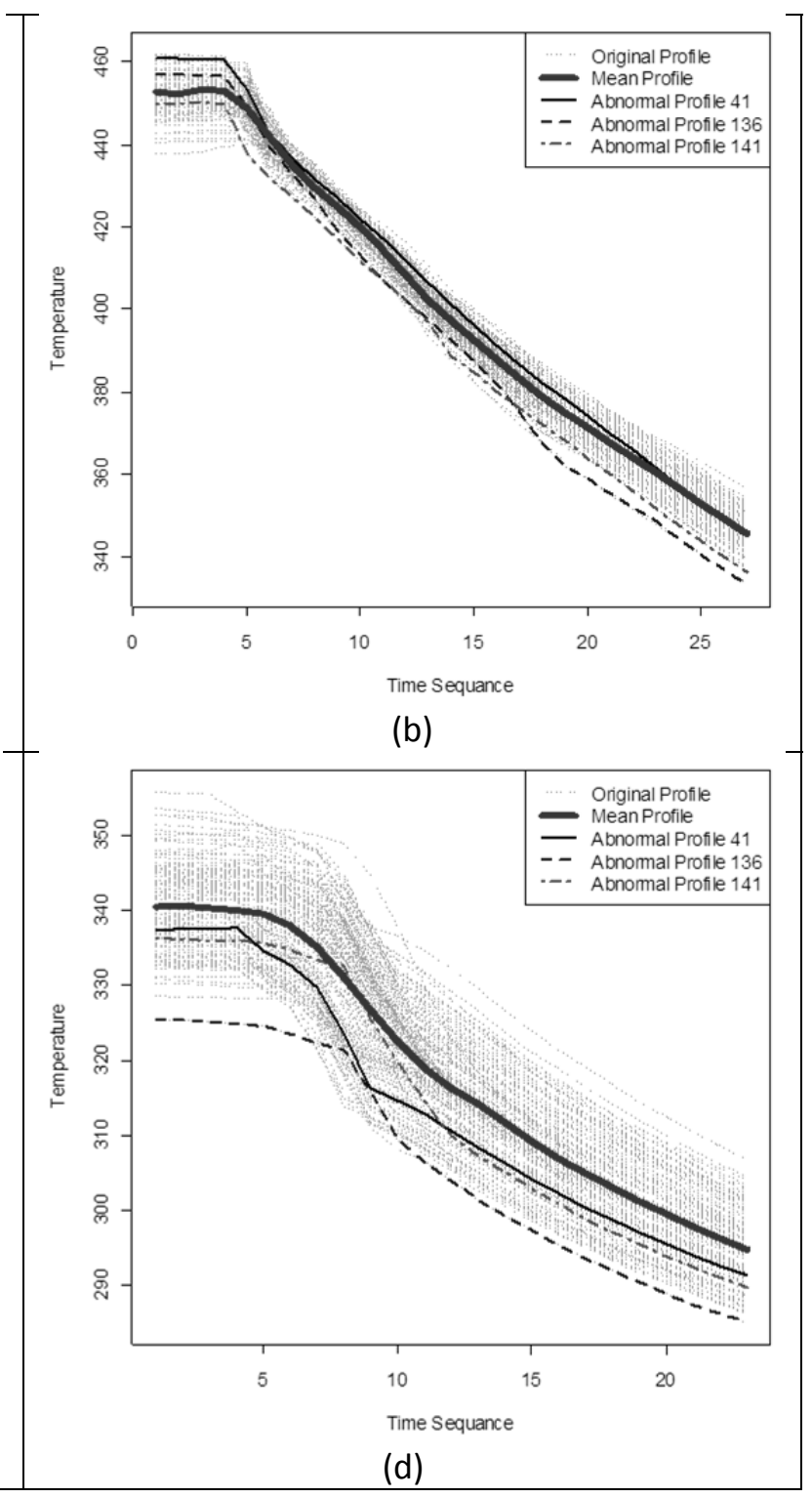

(d)

Figure 9. Cool-down Process Profiles by (a) segment $X_{3}$ (b) segment $X_{4}$ (c) segment $X_{5}$, and (d) segment $X_{6}$ (out-of-control profiles $41,136,141$ ) 

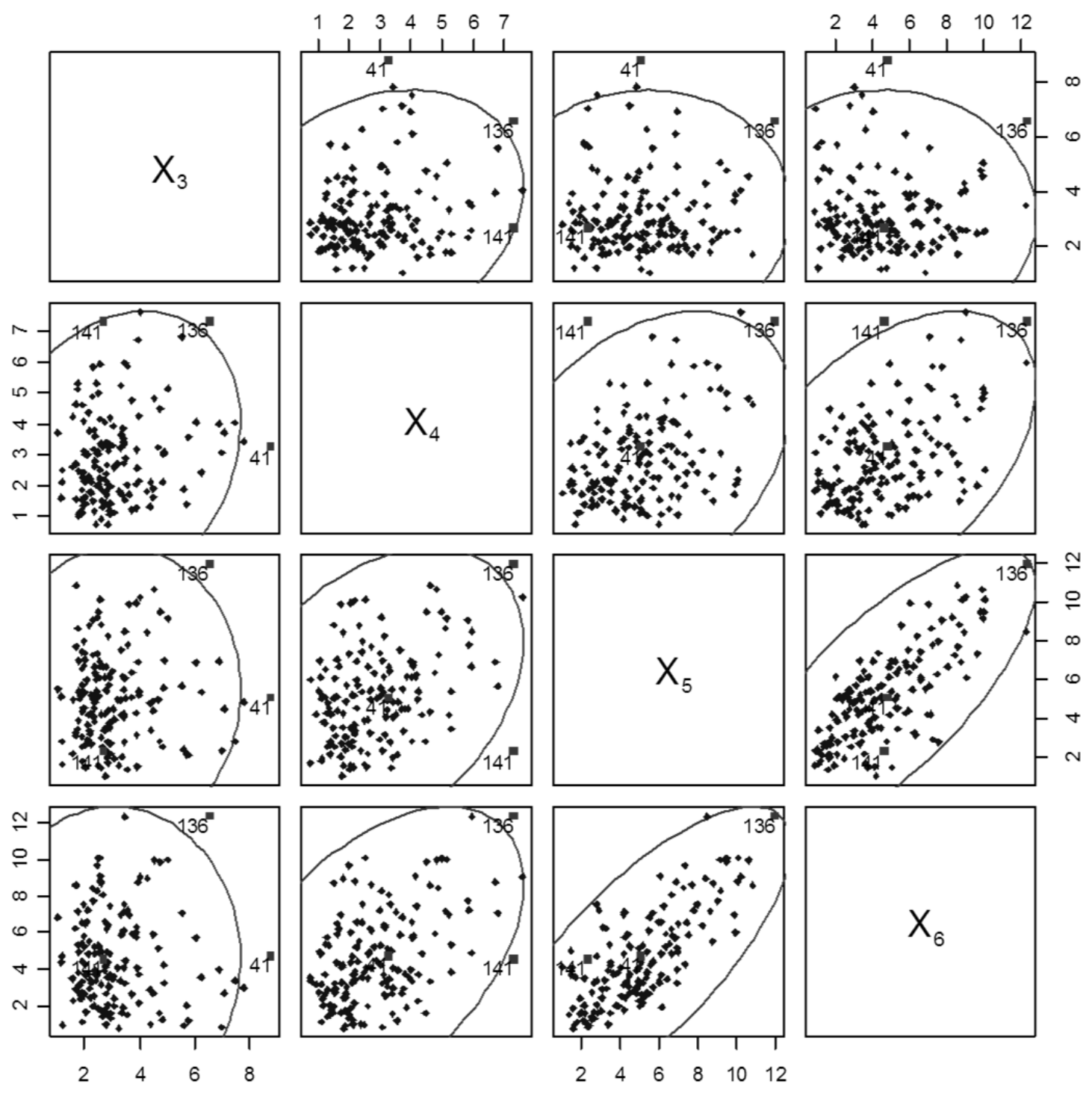

Figure 10. Matrix Plot of Cool-down Variables $X_{3}$ to $X_{6}$ 


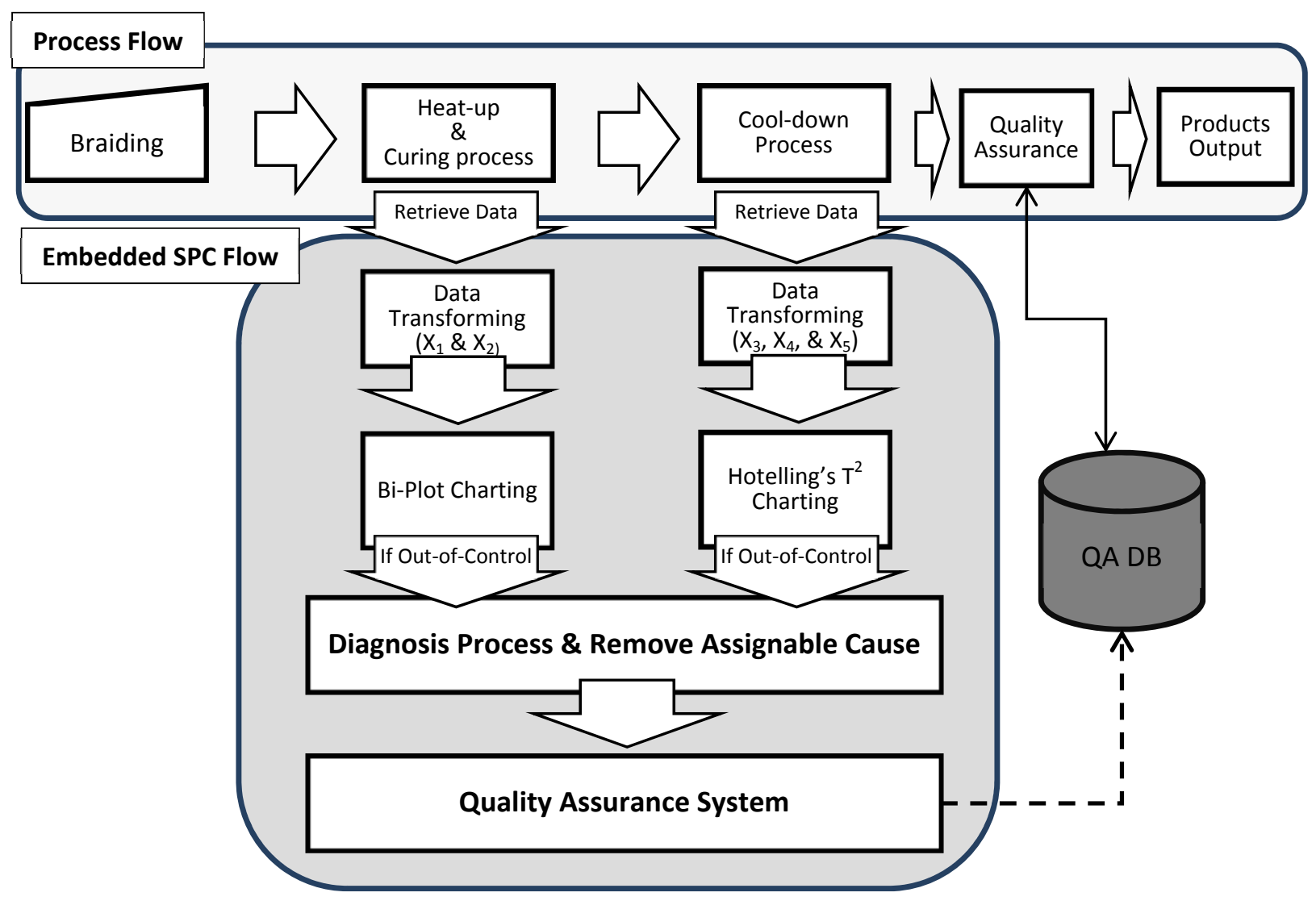

Figure 11. Diagram of Current Process Flow and the Proposed Embedded SPC Flow. 